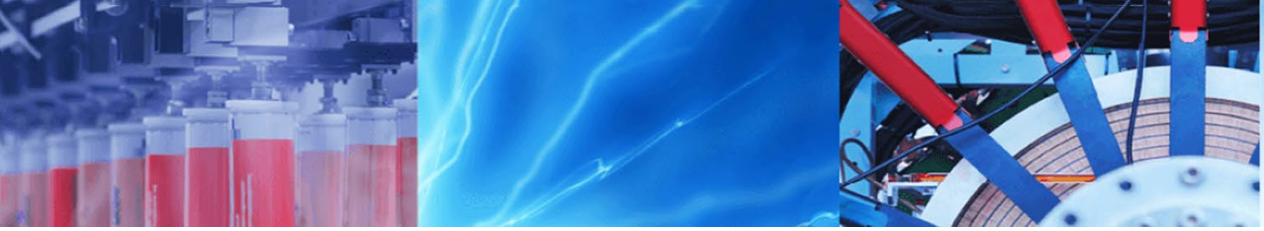

Research Article

\title{
Improvement of scratch resistance of plastic substrates for display screens by employment of low-modulus sublayer
}

\author{
MinGi Kim ${ }^{1}$ Jeong Ho An ${ }^{1}$
}

Received: 17 April 2019 / Accepted: 14 October 2019 / Published online: 24 October 2019

(c) Springer Nature Switzerland AG 2019

\begin{abstract}
Indentation, scratch and steel wool tests were performed on the hard coating surface of multilayered plastic substrate with a soft sublayer. There have been no studies on the effect of soft sublayers on the surface characteristics of plastic substrate. A series of three-layered film composed of a hard coating, plastic substrate and soft sublayer was prepared. In an effort to evaluate the properties of multilayered plastic substrate, including surface, mechanical properties and their resistance to scratches and wear, a comprehensive range of experiments was conducted. Some of the multilayered plastic substrates were found to have excellent scratch resistance while maintaining surface hardness. This is in contrast to many studies in which the improvement of the surface hard coating results in a detrimental effect on scratch resistance. The possibility of improving scratch resistance without scarifying the surface hardness may provide an excellent opportunity to make plastic-based cover screens for mobile devices.
\end{abstract}

Keywords Multilayered plastic substrates · Soft sublayer · Screens for mobile device · Indentation · Surface hardness . Scratch resistance

\section{Introduction}

The proliferation of electronic devices in our lives has driven research into flat-panel displays that are smaller in size, lighter in weight, more rugged and more cost-effective than previous designs. In the smartphone industry, Gorilla Glass has become a standard feature on every mobile phone. It has become a must to avoid scratches, increase durability and give mobile phone a longer life span. On the other hand, plastic has obvious advantages such as lighter weight and more cost-effectiveness than the glass counterpart. In particular, the low cost of plastic plate may become more advantageous as the life span of cell phones decreases and mid-range or low-priced cell phones become more popular in the market. Still, the replacement of Gorilla Glass with a plastic alternative requires overcoming several obstacles including the improvement of surface hardness and scratch resistance.
In order to improve the surface hardness, scratch resistance and abrasion resistance of plastic films, research has been actively conducted on the development of organic-inorganic hybrid hard coating solutions using a sol-gel process or surface treatment techniques such as vacuum deposition. When a hard coating is applied, the surface hardness is generally improved, but the scratch resistance and abrasion resistance properties are not often linearly increased. In particular, the addition of an inorganic filler such as nano-silica improves properties such as surface hardness and heat resistance, but scratch resistance has not been greatly improved or remains marginal [1-3].

Thus, it is difficult to achieve the properties required by conventional methods. Therefore, in this experiment, we studied a method of improving mechanical behavior and scratch resistance by designing the interlayer structure of

MinGi Kim, eok2001@hanmail.net|'Department of Polymer Science and Engineering, SungKyunKwan University, Suwon-Si, Gyeonggi-do 16419, Republic of Korea. 
the materials constituting the plastic substrate in parallel with conventional methods.

In particular, the improvement of scratch resistance of the plastic substrate was achieved by introducing a soft layer on the opposite side of the hard coating surface of the plastic substrate. The soft layer is composed of a thermally cross-linked silicon compound. The cross-linked silicon layer is viscoelastic. Therefore, it can be expected that the viscous nature of the cross-linked layer will provide a damping characteristic which absorbs and dissipates part of the external impact. At the same time, the elastic component of the soft layer may reduce the normal force to improve scratch resistance and help to recover to the original dimensions after the scratching event. However, the introduction of a soft sublayer below the plastic substrate may affect the surface hardness in a bad direction, which was our major concern before the experimental verification.

Nano-indentation and scratch tests for polymer coatings are known to be effective in determining the nanomechanical properties of materials and surface properties [4-11]. In general, the material properties of the thin film need to be measured within $10-20 \%$ of the total film thickness to reduce the influence of the substrate and to maintain the plastic deformation region in the film [12, 13]. For this purpose, nanoscale instruments that can make very shallow indentations should be able to measure a sensitive force of $1 \mathrm{nN}$ and displacement of less than $1 \mathrm{~nm}$ [14-16]. However, to evaluate the characteristics of the entire multilayered plastic substrate in this experiment, a load was applied so that the depth of the indentation was maximized within the range in which the plastic substrate could withstand the strain fracture by stress.

In this study, a series of multilayered plastic substrate with a complex structure consisting of a plastic film between a hard coating layer with high surface hardness on the top and a soft layer on the bottom were fabricated to evaluate mechanical behavior and scratch resistance. In general, most literature focuses on studying the variation of scratch resistance of the substrate either by the compositional change of the substrate or by applying different types of hard coatings [2]. There have been no studies on the effect of a soft sublayer on the surface characteristics of plastic substrate. In this study, we designed a three-layered film composed of a hard coating, plastic substrate and soft sublayer to evaluate the surface characteristics of the composite film. Three different types of plastic substrate with different moduli were used, as well as different thicknesses: $10 \mu \mathrm{m}, 20 \mu \mathrm{m}, 30 \mu \mathrm{m}$ and $40 \mu \mathrm{m}$, to the underlying soft sublayer.

The surface damage characteristics can be evaluated by MAR characteristics representing repeated life scratches and progressive wear which occur on a large area by sweeping and wiping several times with cloth, fabric or tissue. The steel wool test belongs to this category. It is also represented by scratch characteristics, which are one-time scratch due to sharp objects such as a fingernail or key. The pencil hardness test is an example.

The pencil hardness test is a measure of how resistant to fracture a coating film is due to friction from a sharp object. The underlying idea of the test is that an object made of a hard material will scratch an object made of a softer material. On the other hand, the steel wool test is a better method for measuring the progressive wear of a coating film due to repetitive scratches in everyday life. In general, marring occurs under less severe conditions compared to scratching, and the depth of mar damage is less than that of scratches. The distinction between scratching and marring is perhaps best related to appearance attributes. For example, marring is often associated with a high density of small, shallow scratches distributed over a relatively large area such that larger-scale appearance attributes (e.g., gloss or distinctness of image) are affected. Scratching, on the other hand, is associated with a much lower density of larger, deeper scratches, sometimes even a single scratch, where the extent of the scratch dimensions qualitatively depends on a customer's perception of what he or she considers to be a defect.

Scratch and mar resistance are most often characterized using appearance metrics. Currently, however, the relationship between appearance attributes and surface deformation associated with scratching and marring is ambiguous. This lack of connectivity is one of the major barriers to the development and acceptance of a unified standard or set of standards for determining scratch and mar resistance. Another major barrier is the specification of testing conditions. Test variables such as tip geometry, scratch speed and applied load differ from one study to another, sometimes by orders of magnitude. These large variations are even associated with studies in which relatively similar single-probe devices have been used. Because scratch and mar behavior are related to both the surface properties of the material and the loading conditions, laboratory testing should provide an understanding of how the material will perform in a wide variety of conditions. In particular, the effects of temperature, strain and strain rate, which are related to material properties, probe geometry, loading rate and scratch speed, should be thoroughly investigated [17].

To date, only Briscoe et al. [18-22] and more recently Sue and coworkers $[23,24]$ and Gauthier et al. $[25,26]$ have studied the effects of different test variables on the scratch behavior of polymers. In the characterization of mar and scratch resistance of coatings with single-probe techniques, the probe usually scrapes the tested surface under a constant load or increasing load, followed by 
dimensional measurements of the scratch, identification of the critical force at which cracking and fracture occurs, or evaluation with optical instruments [27].

\section{Materials and methods}

The specimens are multilayered films composed of a hard coating on the top, base substrate and soft sublayer.

As a base substrate, the three kinds of base film with different hardnesses/moduli, 150- $\mu$ m-thick PMMA (AF00, Spolytech Co., Ltd., Flexural Modulus 3.3 GPa), 125- $\mu$ m-thick PET (TPL 123, Toray Advanced Material Korea Inc., Flexural Modulus $2.3 \mathrm{GPa}$ ), and 100- $\mu \mathrm{m}$-thick TPU (JTC-1008, Shore Hardness 95A, manufactured by Jin Heung Industrial Co., Ltd., Flexural Modulus $0.003 \mathrm{GPa}$ ) were used. In one series of experiments to evaluate the effect of the base substrate thickness, TPU films with four different thicknesses, $50 \mu \mathrm{m}, 100 \mu \mathrm{m}, 125 \mu \mathrm{m}$ and $200 \mu \mathrm{m}$, were used. TPU films are made of the same material (JTC1008, Shore Hardness 95A, Jin Heung Industrial Co., Ltd.).

As a soft sublayer, cross-linked silicon compound was introduced onto the back side of the base substrate. To evaluate the effect of soft sublayer thickness, the thickness was adjusted into $10 \mu \mathrm{m}, 20 \mu \mathrm{m}, 30 \mu \mathrm{m}$ and $40 \mu \mathrm{m}$, respectively. Also, in order to provide surface hardness to the multilayered plastic substrate, a hard coating solution was applied to the base substrate on the opposite side of the soft sublayer.

The hard coating solution used in this experiment was a 2:1 mixture of hard-type coating solution and softtype coating solution. The hard-type coating solution is an organic-inorganic hybrid type (JHC-008, IOK Co. Ltd.) which provides hardness to the surface, while the softtype coating solution is an organic-type solution (Optoclear 4700, SDM Co., Ltd.) to prevent cracks after curing.

The organic-type coating solution (Optoclear 4700, SDM Co., Ltd.) is composed of $55 \%$ urethane acrylate with a molecular weight of $7500,5 \%$ Irgacure $184,30 \%$ methyl cellosolve and $10 \%$ butyl alcohol. Here, urethane acrylate is composed of hexamethylene diisocyanate (HDI) trimer and pentaerythritol triacrylate (PETA) at a ratio of 2:8. The organic-inorganic hybrid coating solution (JHC-008, IOK Co., Ltd.) is composed of $20 \%$ nano-silica, $10 \%$ pentaerythritol triacrylate (PETA, 10\% methyl methacrylate, $20 \%$ hardener and leveling agent and 40\% 1-methoxy-2-propanol.

The hard coating film was formed by applying the above-mixed hard coating solution with a solid content of $60 \%$ to a plastic substrate using bar coater No. 12. The coating solution applied to the plastic substrate was dried at $90^{\circ} \mathrm{C}$ for $1 \mathrm{~min}$ to remove the solvent, and UV-cured at a light amount of $600 \mathrm{~mJ}$ to form a hard coating film with a uniform thickness.
The soft sublayer was prepared by mixing two silicone pressure-sensitive adhesives (SC3300L and SG6500A, KCC Corporation) at a weight ratio of 50:50 and adding 1 part of a curing agent (SK0010C, KCC Corporation), 1 part of a cross-linking agent (SC0016B, KCC Corporation), and 1 part of a platinum catalyst mixture (SH0003A, KCC Corporation). The soft sublayer with a dried thickness of 10-20 $\mu \mathrm{m}$ was made using bar coaters No. 24 to No. 36 . The soft sublayer with a dried thickness of $30-40 \mu \mathrm{m}$ was made using 4-side applicator. An appropriate amount of silicone adhesive solution was applied on the top of the plastic substrate. The coated film was then cured at $120^{\circ} \mathrm{C}$ for $2 \mathrm{~min}$ and further cured at $160^{\circ} \mathrm{C}$ for $2 \mathrm{~min}$.

The mechanical properties of multilayered plastic substrate were analyzed using a commercially available indenter (Ultra Nanoindentation Tester, Anton-Paar $\mathrm{GmbH}$ ). The measurements were taken by adding a linear load of $95.0 \mathrm{mN}$ for $60 \mathrm{~s}$ to the specimen with a Berkovich indenter according to the Oliver \& Pharr method [28]. To evaluate the characteristics of the entire multilayered plastic substrate, a load was applied so that the depth of the indentation was maximized within the range in which the plastic substrate could withstand the strain fracture by stress.

In the relationship between the stress and strain of the sample when sinusoidal stress is applied to the viscoelastic polymer substance, the viscous part of the viscoelastic polymer material does not respond to a given external force in time, and the viscous response appears with a time lag as a strain by appearing later than the stress. Generally, in a viscoelastic polymer material, the phase difference between stress and strain is $0<\delta<90^{\circ}$. And the elastic stress is in phase with strain and viscous stress is out of phase with strain and is the same as the strain rate phase. Such phase difference is denoted by $\tan \delta$. That is, the larger $\tan \delta$ of the viscoelastic polymer means that the damping capacity inside the sample becomes larger [29].

$\tan \delta=E^{\prime \prime} / E^{\prime}$ (A criterion of the damping ability of a substance)

In order to measure the scratch characteristics and elastic recovery of multilayered plastic substrate, a commercial indenter (Micro Scratch Tester (MST), Anton-Paar GmbH) was employed. The scratch characteristics were measured by increasing the load at a speed of $233 \mathrm{mN} / \mathrm{s}$ until the end load reached $7000 \mathrm{mN}$, starting from a $10-\mathrm{mN}$ initial load for the progressive type indenter. The moving speed of the tip was $12 \mathrm{~mm} / \mathrm{min}$, and the scratch length was $6 \mathrm{~mm}$. Immediately after scratching, the scratch depth was rescanned with a scanning load of $10 \mathrm{mN}$ using the same tip to measure the residual indented depth.

An indenter that is a Rockwell-type diamond tip (Al-200) with a radius of $200 \mu \mathrm{m}$ and angle of $120^{\circ}$ was used in the experiment. The tip is shown in Fig. 1. This procedure 


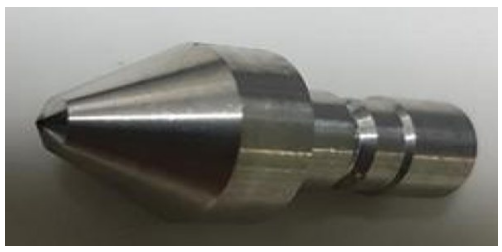

Fig. 1 Rockwell-type diamond indenter tip (Al-200: $200 \mu \mathrm{m}$ radius and angle of $120^{\circ}$ )

enables the evaluation of the elastic recovery of the multilayered plastic substrate. Also, the scratch characteristics of the multilayered plastic substrate with a soft sublayer were evaluated according to the variation of the soft sublayer thickness.

For the evaluation of surface roughness, root-meansquare (RMS) roughness values were measured by using AFM (ToscaTM, Anton-Paar GmbH). Measurements were taken at $0.125-\mu \mathrm{m}$ intervals for each axis at a size of $50 \mu \mathrm{m}$ on the horizontal axis and $50 \mu \mathrm{m}$ on the vertical axis of the sample.

The film thickness of each layer of the multilayered plastic substrate including the hard coating layer and the soft sublayer was measured using an SEM (FE-SEM S-4700, Hitachi).

In order to measure the friction and wear characteristics, scratch characteristics were evaluated using an Abrasion Rubbing Tester (No. 580, Yuyuinst Co., Ltd.). The scratch characteristics were evaluated by measuring the number of rubbing cycles until a scratch was generated in the hard coating film by applying a load of $1 \mathrm{~kg}$ to a bar with steel wool (\#0000) attached thereto. At this time, the bar reciprocates the moving distance of $40 \mathrm{~mm}$ at a moving speed of 80 RPM. After the scrapings, images of the scratches were taken by optical microscope (GX51F, Olympus). The scratch examples can be seen through Fig. 2 .
A conventional pencil hardness test was also performed according to ASTM D 3363 on the multilayered plastic substrate using a pencil hardness tester (CT-PC2, Coretech Korea Co., Ltd.). The hardnesses were evaluated by measuring the pencil hardness value at the time of occurrence of a scratch by repeatedly measuring five times under each hardness condition by applying a load of $1 \mathrm{~kg}$ to pencils with a different hardness. At each measurement, the pencil moves a distance of $20 \mathrm{~mm}$ at a moving speed of $120 \mathrm{~mm} / \mathrm{min}$. This is a qualitative value that depends on the hardness of the pencil used and is not a quantitative evaluation method capable of evaluating accurate scratch characteristics.

\section{Results and discussion}

The overall structure of multilayered film is shown in Fig. 3, where the plastic substrate is sandwiched between a hard coating layer and soft sublayer. To confirm the thickness variation of the soft sublayer, the thickness of each layer of the multilayered plastic film was observed by SEM, as shown in Fig. 4. The hard coating and soft sublayer are applied to PMMA film that is $150 \mu \mathrm{m}$ thick. It can be confirmed that the thickness of

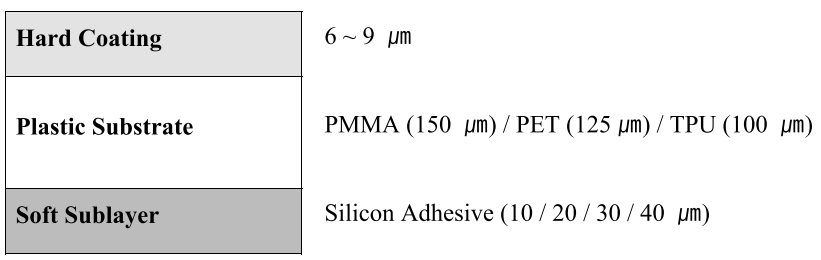

Fig. 3 Structural diagram of multilayer plastic substrate
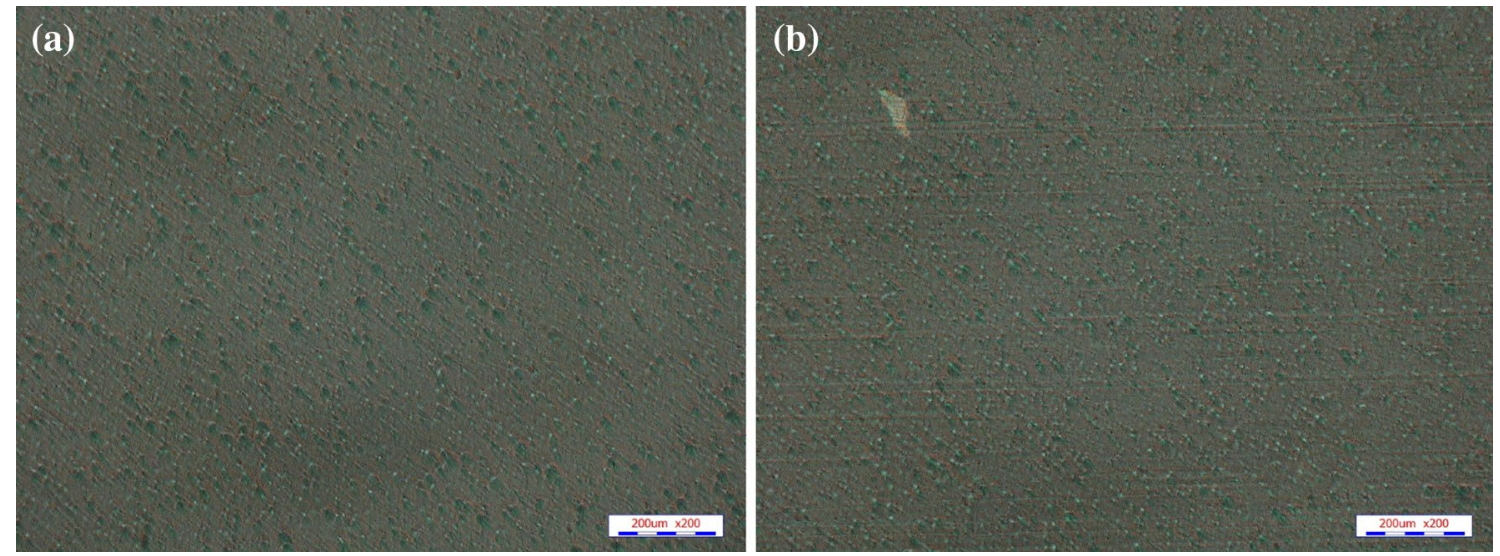

Fig. 2 Optical microscope images of the hard coating surface of multilayered plastic substrate in the process of the abrasion test. a The hard coating surface before scratches occurred, $\mathbf{b}$ The hard coating surface at the time scratches occurred 


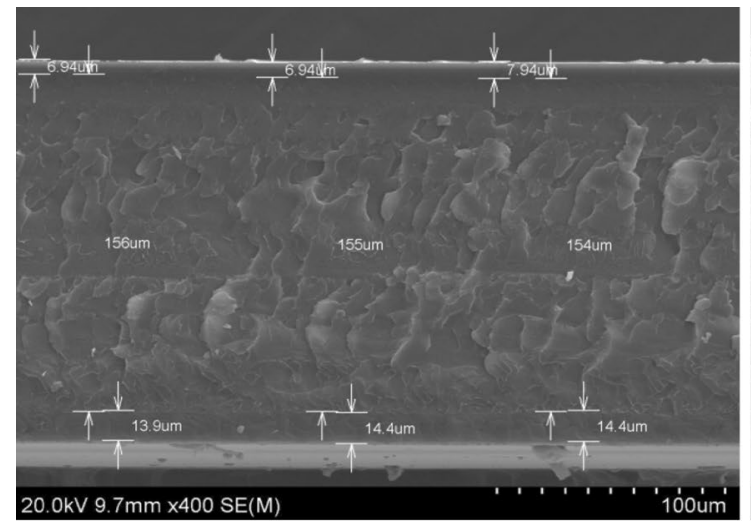

(a) $10 \mu \mathrm{m}$ Soft Sublayer

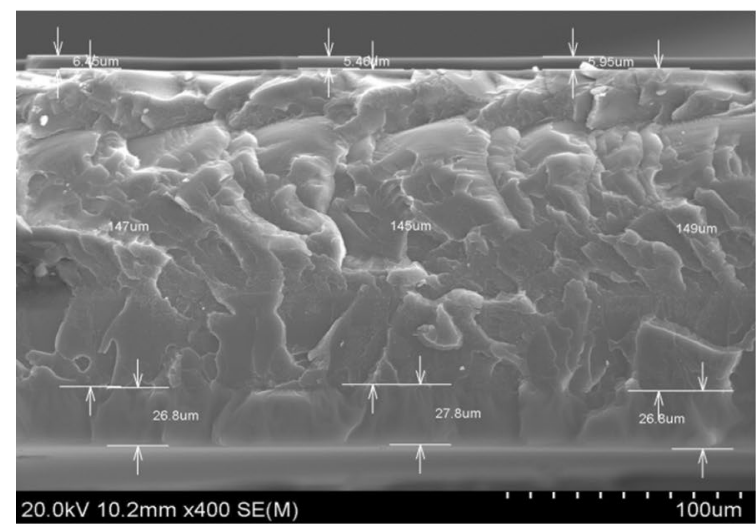

(c) $30 \mu \mathrm{m}$ Soft Sublayer

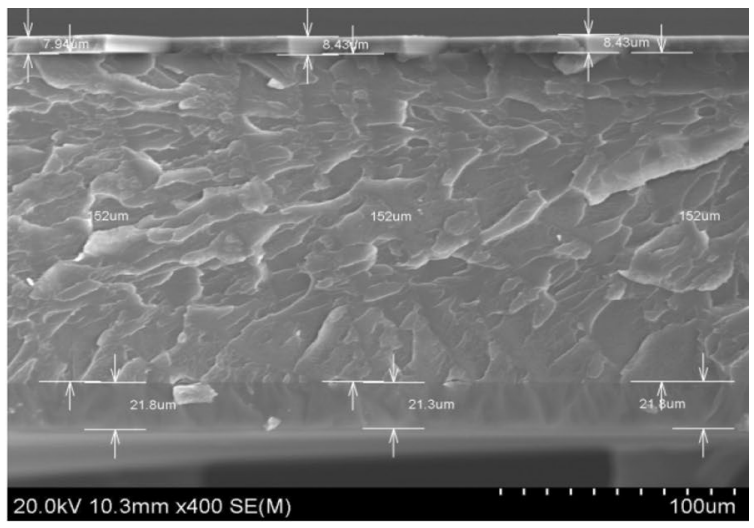

(b) $20 \mu \mathrm{m}$ Soft Sublayer

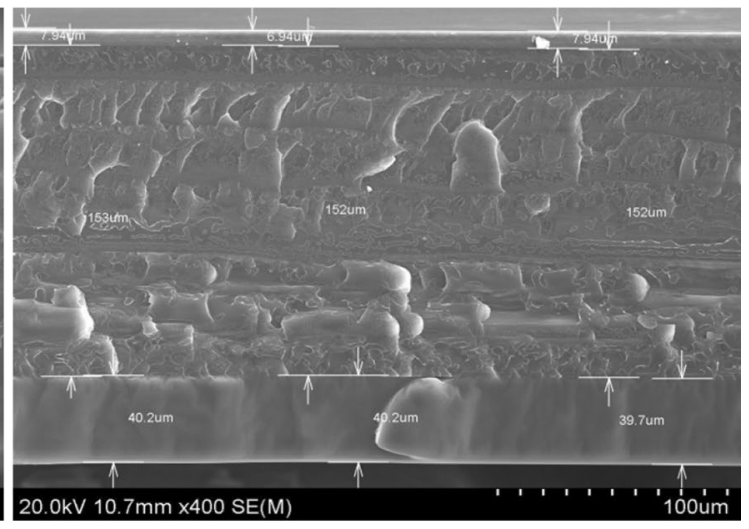

(d) $40 \mu \mathrm{m}$ Soft Sublayer

Fig. 4 Typical SEM image of multilayered plastic substrate. The current film is composed of a hard layer (on top), 150- $\mu \mathrm{m}$ PMMA film (in the middle), and a soft sublayer (on the bottom)

soft sublayer varied from 10 to $40 \mu \mathrm{m}$ as intended, and the thickness of the hard coating is around 6-9 $\mu \mathrm{m}$.

Figure 5 shows AFM surface images of the hardcoated side of multilayered plastic substrate in Fig. 4, and the measured root-mean-square (RMS) roughness values are summarized in Table 1. The surface roughness values of four kinds of multilayered plastic substrate were all in the range of $1.66-1.73 \mathrm{~nm}$, indicating the uniformity of the hard coating. Uniformity of surface roughness among samples is very important since roughness is the one of the most important factors in determining wear or frictional properties. The RMS roughness values of the four samples were very low, thus indicating that the hard coating surface is very smooth and uniform. Therefore, among the two factors that influence the frictional force in the scratch test, namely normal force and coefficient of friction, we can safely assume that the coefficient of friction of the hard coating film surface was very similar among the samples. Therefore, normal force is the main factor in determining the scratch properties of multilayered film.
A soft sublayer was introduced onto one side of a PMMA film with a thickness of $150 \mu \mathrm{m}$ at thicknesses of $10 \mu \mathrm{m}$, $20 \mu \mathrm{m}, 30 \mu \mathrm{m}$ and $40 \mu \mathrm{m}$, respectively. Then, four types of multilayered plastic substrate on which the hard coating film was formed with the same material and curing conditions on the opposite surface to which the soft sublayer was introduced were made. In order to measure the friction, abrasion and elastic recovery rate of the hard coating film of these substrates, scratch characteristics were evaluated using a commercial indenter (Micro Scratch Tester (MST), Anton-Parr GmbH) and are shown in Tables 2 and 3.

Permanent indentation occurred at a stress of $2000 \mathrm{mN}$ for the film with the least thick soft sublayer, while the one with the thickest soft sublayer, $40 \mu \mathrm{m}$, withstood indentation stress up to $5600 \mathrm{mN}$. Since the hard coating layer is the most brittle layer among the layers and has the least elastic limit, the permanent indentation is considered to be initiated from the top hard coating layer. Within the range in which the hard coating layer of plastic substrate can withstand the strain fracture by stress, as the thickness of the soft sublayer increases, the critical stress increases. 


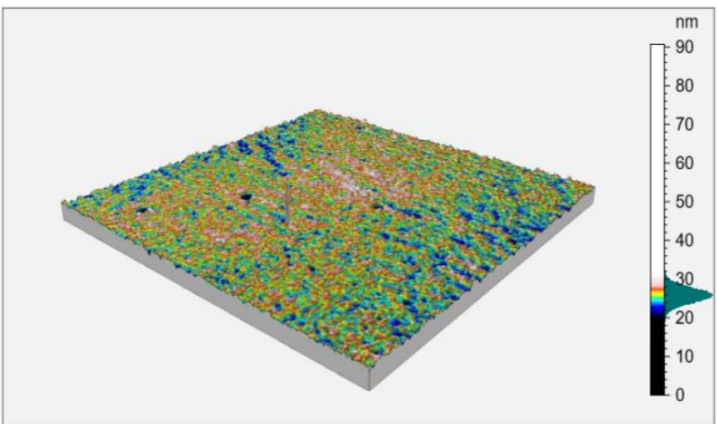

(a) $10 \mu \mathrm{m}$ Soft Sublayer

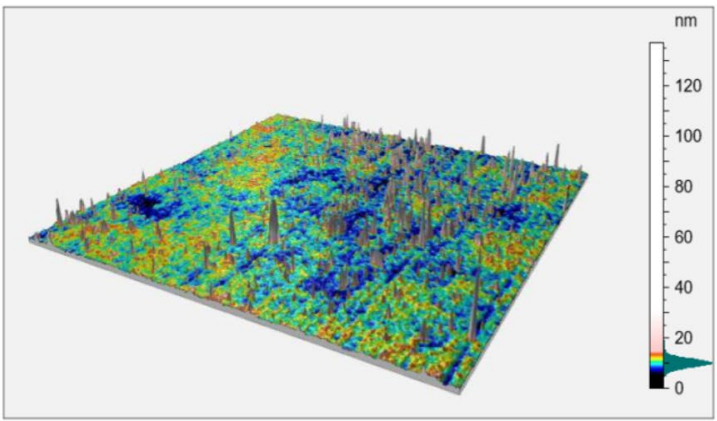

(c) $30 \mu \mathrm{m}$ Soft Sublayer

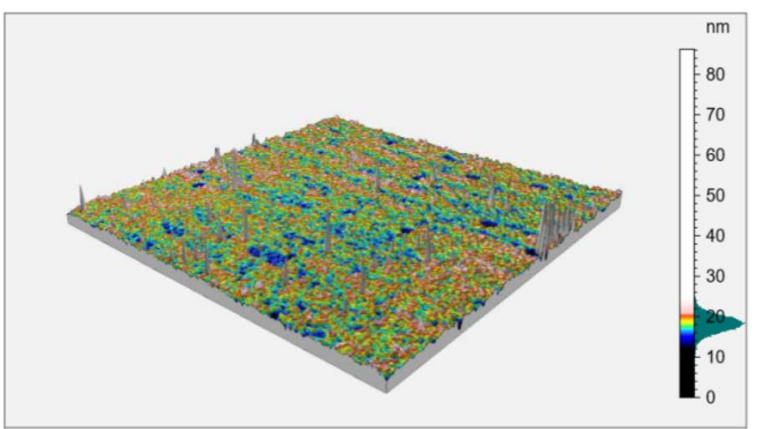

(b) $20 \mu \mathrm{m}$ Soft Sublayer

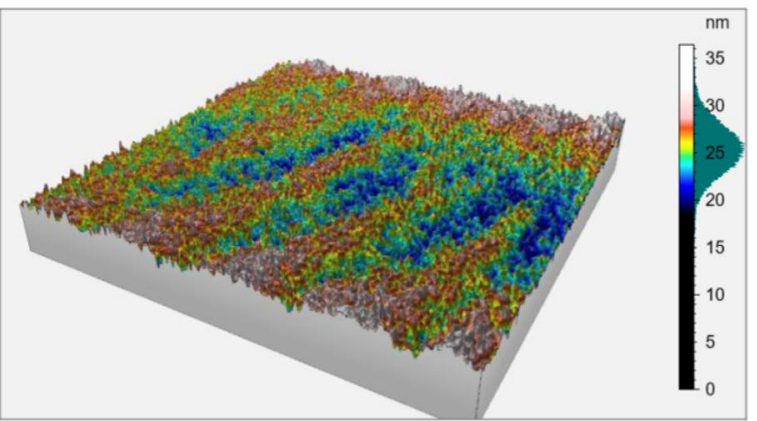

(d) $40 \mu \mathrm{m}$ Soft Sublayer

Fig. 5 AFM image of hard coating surface of multilayered plastic substrate in Fig. 4

Table 1 Surface roughness of hard coating layer of multilayered plastic substrate. An identical hard coating is applied to $150-\mu \mathrm{m}$ PMMA film

\begin{tabular}{lll}
\hline Sample name & $\begin{array}{l}\text { Hard coating thickness } \\
(\mu \mathrm{m})\end{array}$ & $\begin{array}{l}\text { RMS } \\
\text { roughness } \\
(\mathrm{nm})\end{array}$ \\
\hline $10-\mu \mathrm{m}$ soft Sublayer & 7.3 & 1.70 \\
$20-\mu$ m soft sublayer & 8.3 & 1.66 \\
$30-\mu \mathrm{m}$ soft sublayer & 6.0 & 1.72 \\
$40-\mu$ m soft sublayer & 7.6 & 1.73 \\
\hline
\end{tabular}

Table 2 The critical stress (minimum stress for permanent indentation) for hard-coated PMMA substrate $(150 \mu \mathrm{m})$ with four different soft sublayers with thicknesses of $10 \mu \mathrm{m}, 20 \mu \mathrm{m}, 30 \mu \mathrm{m}, 40 \mu \mathrm{m}$

\begin{tabular}{lllll}
\hline & \multicolumn{4}{l}{ Soft sublayer } \\
\cline { 2 - 5 } & $10 \mu \mathrm{m}$ & $20 \mu \mathrm{m}$ & $30 \mu \mathrm{m}$ & $40 \mu \mathrm{m}$ \\
\hline Stress $(\mathrm{mN})$ & 1994.322 & 2492.374 & 5470.648 & 5578.492 \\
\hline
\end{tabular}

Therefore, the deformation of the specimen in the depth direction increases with the increased thickness of the soft sublayer so that it can withstand more stress.

The above behavior is also confirmed by the peak indentation values given in Table 3 . Since the applied force
Table 3 Peak indentation depth, residual indentation depth and elastic recovery rate (\%) of each sample when the same load (a load of $1994 \mathrm{mN})$ is applied to a hard-coated PMMA substrate $(150 \mu \mathrm{m})$ with different thicknesses of soft sublayer

\begin{tabular}{llcl}
\hline $\begin{array}{l}\text { Soft sublayer } \\
\text { thickness }(\mu \mathrm{m})\end{array}$ & $\begin{array}{l}\text { Peak indenta- } \\
\text { tion depth } \\
(\mathrm{nm})\end{array}$ & $\begin{array}{l}\text { Residual indenta- } \\
\text { tion depth }(\mathrm{nm})\end{array}$ & $\begin{array}{l}\text { Elastic recov- } \\
\text { ery rate }(\%)^{*}\end{array}$ \\
\hline 10 & 13,180 & 1023 & 92.24 \\
20 & 14,702 & 716 & 95.13 \\
30 & 15,539 & 619 & 96.02 \\
40 & 16,186 & 422 & 97.39 \\
\hline
\end{tabular}

* Elastic recovery rate $(\%)=\frac{\text { Peak indentation depth }- \text { residual indentation depth }}{\text { Peak indentation depth }} \times 100$

is $1994 \mathrm{mN}$, it is below the critical stress for the specimen with a soft sublayer thicker than $10 \mu \mathrm{m}$. The peak indentation depth increased as the thicker soft sublayer was applied. For residual indentation, the specimen with a $10-\mu \mathrm{m}$ soft sublayer showed the highest value, and much lower values were observed for specimens with a thicker soft sublayer. Considering that the thickness of the hard coating layer is around $8000 \mathrm{~nm}$, the residual indentation depth of $500 \mathrm{~nm}$ indicates that minimal or negligible cracks are formed on the hard-coated surface. Similar behavior was observed by Pierre Morel and Vincent Jardret 
[30]. In this research, a PMMA sheet without any hard coating or soft sublayer was tested with the indenter, while the measurement was taken under varying temperatures. The critical load increased with increasing temperature, with an inflection point around $45^{\circ} \mathrm{C}$, which is near the brittle/ ductile transition in a uniaxial tensile test. This indicates that the increasing ductility of a specimen can result in the increase in critical load.

The damping characteristics of plastic layers below the hard coating seem to play a certain role in increasing critical load. The viscoelastic nature of plastic may absorb part of the deformation energy supplied by the indentation tip, and such energy dissipation becomes more effective with a thicker soft sublayer, resulting in the increased critical load. Since the soft sublayer is composed of cross-linked silicon, it will have an elastic character to help recovery to the original dimensions. This tendency is well reflected in the decreased residual indentation and elastic recovery rate in Table 3, where the residual indentation depth decreases from 1023 to $422 \mathrm{~nm}$ and the elastic recovery rate increases from 92 to $97 \%$ with the increasing thickness of the soft sublayer.

In order to compare the hardness and modulus of the hard-coated PMMA with different thicknesses of soft sublayer, the mechanical properties were evaluated using a commercial indenter (Ultra Nanoindentation Tester, Anton-Paar $\mathrm{GmbH}$ ), and the results are shown in Table 4. In this experiment, to evaluate the characteristics of the entire multilayered plastic substrate, a load of $95 \mathrm{mN}$ was applied so that none of the three layers were subject to strain fracture.

The surface hardness $\mathrm{H}_{\text {IT }}$ of multilayered film was $392 \mathrm{MPa}$ when the thickness of the soft sublayer was $10 \mu \mathrm{m}$ and was $377 \mathrm{MPa}$ with a $40-\mu \mathrm{m}$-thick soft sublayer. The surface hardness of the multilayered film was not affected much by the thickness of the soft sublayer and showed a quite high value in the range of 350-400 MPa. This result is in contrast to a study on PMMA plate only by Jardet et al. [30]. When the temperature of the

Table 4 Mechanical properties measured by adding a linear load of $95.0 \mathrm{mN}$, which was applied to four types of multilayered plastic substrate with a soft sublayer with thicknesses of $10 \mu \mathrm{m}, 20 \mu \mathrm{m}$, $30 \mu \mathrm{m}$ and $40 \mu \mathrm{m}$ with a Berkovich indenter for $60 \mathrm{~s}$

\begin{tabular}{lllll}
\hline & \multicolumn{4}{l}{ Soft sublayer } \\
\cline { 2 - 5 } & $10 \mu \mathrm{m}$ & $20 \mu \mathrm{m}$ & $30 \mu \mathrm{m}$ & $40 \mu \mathrm{m}$ \\
\hline $\mathrm{H}_{\mathrm{IT}}(\mathrm{O} \& \mathrm{P}),(\mathrm{MPa})$ & 392 & 355 & 359 & 377 \\
$\tan \delta$ (Sinus mean) & 0.031 & 0.037 & 0.063 & 0.083 \\
$\begin{array}{l}\text { Storage modulus (Sinus mean), } \\
\quad(\mathrm{GPa})\end{array}$ & 4.593 & 4.322 & 3.716 & 3.794 \\
Loss modulus (Sinus mean), (GPa) & 0.144 & 0.161 & 0.234 & 0.316 \\
\hline
\end{tabular}

measurement was changed from 20 to $80^{\circ} \mathrm{C}$, the storage modulus of PMMA decreased while the indentation hardness of the specimen decreased from $300 \mathrm{MPa}$ to $100 \mathrm{MPa}$, which is quite natural, considering that PMMA becomes softer with increasing temperature. However, when a hard-coated PMMA plate is supported by a soft sublayer, the surface hardness can be maintained with the current construction of multiple layers.

The peak indentation depth increased in proportion to the increase in the soft sublayer thickness (Table 3), but the surface hardness $\mathrm{H}_{1 T}$ was maintained at a similar value regardless of the thickness variation. In other words, if the thickness of the soft sublayer is increased, the entire film is more deformable, but its surface hardness is maintained. The insensitivity of surface hardness on the thickness of the soft sublayer is quite reasonable considering that the major factor determining hardness is the top layer, which is the hard coating layer. The pencil hardness of multilayered plastic substrate showed a similar trend, as seen in Table 5. As shown in Table 5, the entire hard-coated surface of multilayered plastic substrate was scratched at $6 \mathrm{H}$ regardless of the thickness of the soft sublayer. Pencil hardness was not affected by the thickness of the soft sublayer and showed similar surface hardnesses.

Other mechanical characteristics such as $\tan \delta$, storage modulus and loss modulus are also affected by the addition of a soft sublayer. The storage modulus, a component of acting as an elastic repulsive force in multilayered plastic substrate, gradually decreased from 4.6 to $3.7 \mathrm{GPa}$, while the thickness of the soft sublayer increased from 10 to $40 \mu \mathrm{m}$ as a natural consequence of the presence of the soft sublayer. The $\tan \delta$ value, which refers the damping ability of the multilayered film to external stress, was 0.031 at a soft sublayer thickness of $10 \mu \mathrm{m}, 0.063$ at $20 \mu \mathrm{m}, 0.063$ at $30 \mu \mathrm{m}$ and 0.083 at $40 \mu \mathrm{m}$. On the other hand, the loss modulus increased significantly from 0.144 to $0.316 \mathrm{GPa}$ with the increase in thickness of the soft sublayer from 10 to $40 \mu \mathrm{m}$.

In the micro-indentation test, the addition of a soft sublayer is a good way to maintain surface hardness while providing a certain degree of deformability to the entire multilayered film. However, surface hardness and scratch resistance are quite often two different things. In order to test the scratch resistance of the multilayered film, the same specimens were subject to a steel wool test.

As shown in Fig. 3, three different types of plastic substrate, PMMA, PET and TPU, were used, and the same type of hard coating was applied on the top of each plastic substrate. On the bottom side, soft sublayers $0 \mu \mathrm{m}, 10 \mu \mathrm{m}$, $20 \mu \mathrm{m}, 30 \mu \mathrm{m}$ and $40 \mu \mathrm{m}$ thick were formed using crosslinked silicon adhesive.

The results of steel wool test are summarized in Fig. 6 . When there is no soft sublayer, the application of steel 
Table 5 Pencil hardness of multilayered plastic substrate in which a soft sublayer was introduced at thicknesses of $0 \mu \mathrm{m}, 10 \mu \mathrm{m}, 20 \mu \mathrm{m}, 30 \mu \mathrm{m}$, $40 \mu \mathrm{m}$ to an identical hardcoated PMMA substrate (150 $\mu \mathrm{m}$ thick)

\begin{tabular}{|c|c|c|c|c|c|c|}
\hline \multirow{2}{*}{$\begin{array}{l}\text { Measuring condi- } \\
\text { tions } \\
\text { Load }\end{array}$} & \multicolumn{6}{|l|}{ Soft sublayer } \\
\hline & $\begin{array}{l}\text { Pencil hard- } \\
\text { ness }\end{array}$ & $0 \mu \mathrm{m}$ & $10 \mu \mathrm{m}$ & $20 \mu \mathrm{m}$ & $30 \mu \mathrm{m}$ & $40 \mu \mathrm{m}$ \\
\hline \multirow[t]{12}{*}{$1 \mathrm{~kg}$} & $8 \mathrm{H}$ & & & & & \\
\hline & $x$ & 4 & 1 & 2 & 2 & 1 \\
\hline & O & 1 & 4 & 3 & 3 & 4 \\
\hline & $7 \mathrm{H}$ & & & & & \\
\hline & $x$ & 2 & 4 & 3 & 2 & 2 \\
\hline & O & 3 & 1 & 2 & 3 & 3 \\
\hline & $6 \mathrm{H}$ & & & & & \\
\hline & $x$ & 1 & 1 & 1 & 2 & 1 \\
\hline & 0 & 4 & 4 & 4 & 3 & 4 \\
\hline & $5 \mathrm{H}$ & & & & & \\
\hline & $\mathrm{X}$ & - & - & - & - & - \\
\hline & 0 & 5 & 5 & 5 & 5 & 5 \\
\hline
\end{tabular}

$X=$ Scratches, $O=$ No Scratches (Clear)

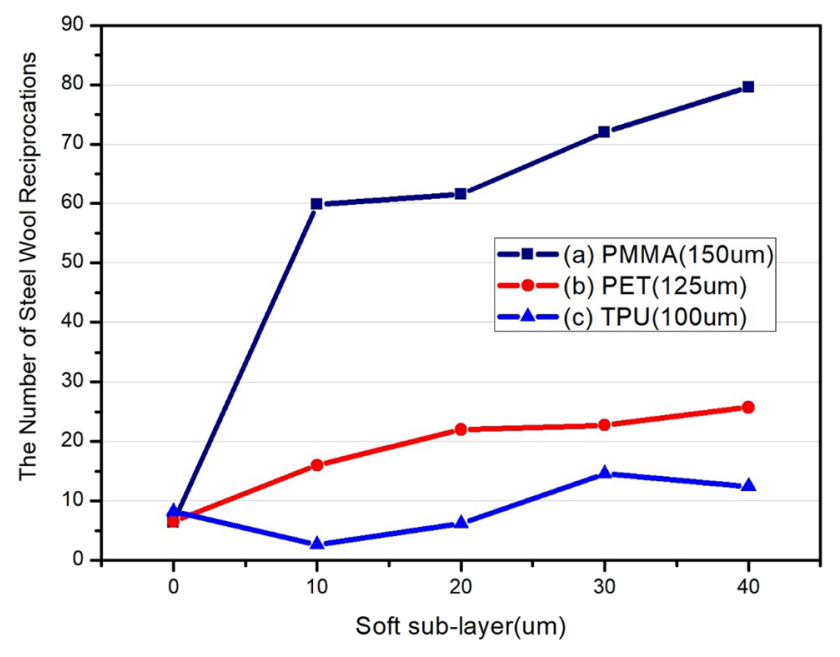

Fig. 6 Critical steel wool reciprocal abrasions for permanent scratches. An identical hard coating was applied for three different types of substrate, while different thicknesses of soft sublayer were used. Each experiment was conducted at 5 times. Standard deviations are as follows: a $1.30,0.55,0.45,0.89,1.82 ; \mathbf{b} 1.29,0.82,0.82$, $0.96,1.50 ;$ c $1.14,0.84,0.89,1.00,1.52$

wool reciprocal abrasions only five to eight times was enough to cause permanent scratches depending on the kind of plastic substrate used. This suggests that the use of hard coating is good to increase surface hardness as shown in the indentation test, but is of no use to improve scratch resistance.

If the thickness of the soft sublayer is increased, the minimum number of steel wool reciprocal abrasions to induce surface scratches generally increases with the increase in soft sublayer thickness. While the minimum number of steel wool reciprocal abrasions is five for the one without a soft sublayer, the number increases to 59 with only a 10- $\mu \mathrm{m}$-thick soft sublayer. The composite film becomes more scratch resistant with the increasing thickness of the soft sublayer. The one with a $40-\mu \mathrm{m}$ soft sublayer results in near 80 , which is about 16 times more scratch resistant compared with the one without a soft sublayer.

The effect of adding a soft sublayer could be explained in two different respects. First, it comes from the reduction in normal force by local deformation under the steel wool. There are two factors in determining frictional force, as shown below:

Frictional force $(F)=$ Coefficient of friction $(\mu) \times$ Normal force $(N)$

Here, the coefficient of friction $\mu$ may vary depending on the material of the object, the smoothness of the surface, the presence or absence of lubricant, and so on. However, if the material and surface state of the hard coating surface are similar to each other as in the respective samples used in this experiment, the frictional force is dominantly influenced by the normal force rather than the coefficient of friction. For a hard-coated PMMA film without a soft sublayer, $N=\mathrm{mg}$ because it can be seen as the normal force $(N)$ acting on the horizontal plane (Fig. 7).

On the other hand, in the case of a hard-coated PMMA film with soft sublayer, a gradient occurs under a moving steel wool bar due to the pressing of the soft under layer when a load is applied. And as the thickness of the soft layer becomes thicker, this gradient becomes larger, and the normal force becomes smaller. That is, for a hardcoated PMMA film with a soft under layer, $N=m g \operatorname{Cos} \theta$ because it can be seen as the normal force $(N)$ acting on 


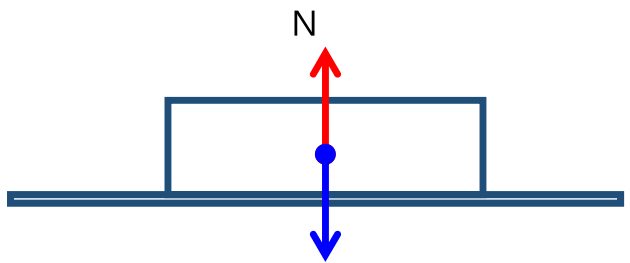

$\mathrm{mg}$

Fig. 7 Normal force $(N)$ acting on the horizontal plane

$\mathrm{N}$

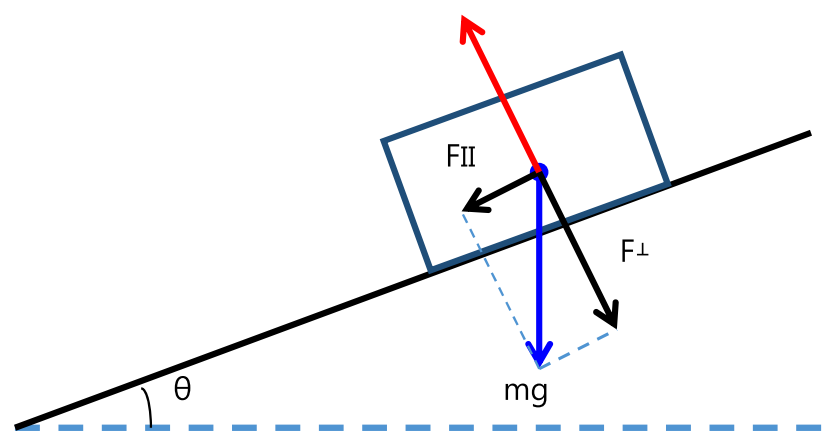

Fig. 8 Normal force $(N)$ acting on the incline

the incline (Fig. 8). Such inclination increases as the thickness of soft sublayer increases.

Second, since the steel wool test has a dynamic nature, it can be assumed that the damping characteristics of the soft sublayer play a certain role. During the steel wool test, the multilayered film is subject to compressional deformation on the front and tensional deformation on the tailing side of the steel wool moving direction. During these deformations, the damping capability of film may absorb and dissipate part of the deformation energy. As shown in the indentation test, as the thickness of soft sublayer is increased, the $\tan \delta$ value becomes larger and more energy can be dissipated to prevent surface scratches.

Such an observation suggests that the improvement of scratch resistance with the employment of the soft sublayer is strongly related to the damping characteristics of the soft sublayer. Since the improvement of surface hardness and scratch resistance originates from two separate factors, which is the hard coating on the top and the soft sublayer on the bottom, there is a window to improve scratch resistance without losing the surface hardness of the film.

When the softer base substrate is used, quite different behaviors are observed. Specimen (b) in Fig. 6 is a multilayered film using a $125-\mu \mathrm{m}$-thick PET film that has a lower modulus than PMMA film does. In this case, steel wool reciprocal abrasions eight times result in permanent scratches without using a soft sublayer. The addition of a soft sublayer has some effect. If the thickness of the soft sublayer is increased, the point of occurrence of scratches moves to reciprocal abrasions 27 times, which is an improvement in scratch resistance of about two to three times. Clearly, the effect of using a soft sublayer diminishes as the modulus of the polymer base film is reduced. Such a tendency becomes more obvious if a softer polymer substrate is used, which is the case with specimen (c) using 100- $\mu$ m-thick TPU film. With no soft sublayer, reciprocal abrasions seven times are enough to cause surface scratches. If the thickness of soft sublayer is increased, reciprocal abrasions 14 times were recorded with a $40-\mu \mathrm{m}$-thick soft layer. Therefore, the improvement is only about two times.

According to the above results, it seems that the modulus of the base film or the modulus ratio of base film and soft sublayer is an important factor in determining the effectiveness of the soft sublayer on improving scratch resistance characterized by the steel wool test. Experimental observation suggests that the higher the hardness of base film, the greater the effectiveness of introducing the soft sublayer for the improvement of scratch resistance tested by the steel wool test. This seems to be the result of the presence of the viscoelastic property of the base substrate itself. When a soft substrate such as TPU film is used, the TPU itself has a damping effect on external stress due to the viscose response of TPU. Therefore, even if a soft sublayer is introduced below the TPU film, the additional damping effect of the soft sublayer becomes relatively small due to the damping effect of the TPU film itself. Moreover, a soft substrate may not provide enough support for a hard coating layer so that the hard coating layer is fractured by too much strain. On the other hand, when a high hardness material such as a PMMA film is used as a base film, the hard PMMA acts as a support layer for preventing deformation (destruction) of the hard coating film due to external stress.

As shown in Fig. 6c, when TPU film was used as a base film, the effect of the soft sublayer addition on scratch resistance was relatively small. This is because the viscous properties of the TPU itself are large. In order to confirm the damping effect of the TPU film itself, the scratch resistance test was performed on hard-coated TPU films with thicknesses of $50 \mu \mathrm{m}, 100 \mu \mathrm{m}, 125 \mu \mathrm{m}$ and $200 \mu \mathrm{m}$ without any soft sublayer. The results are shown in Fig. 9.

When the thickness of the TPU film was $50 \mu \mathrm{m}$ and $100 \mu \mathrm{m}$, the number of steel wool reciprocal abrasions until scratches occurred was six and seven times, not showing much improvement. When the thickness of the TPU film was increased to $125 \mu \mathrm{m}$ and $200 \mu \mathrm{m}$, the number of steel wool reciprocal abrasions was 14 and 15, respectively. The scratch resistance of the TPU substrate with only 


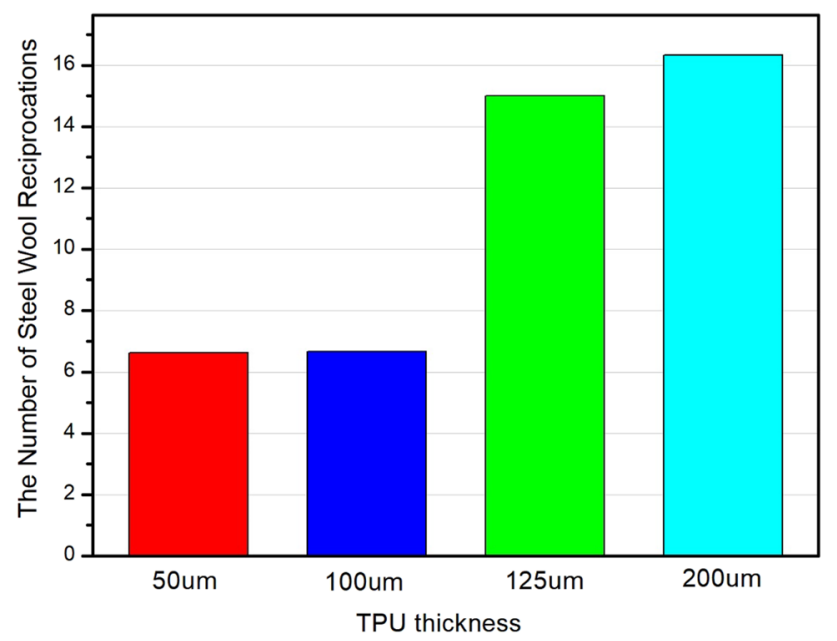

Fig. 9 Scratch resistance test of TPU film without a soft sublayer. Only a hard coating is applied on top of TPU film with different thicknesses. Each experiment was conducted at three times. Standard deviations are as follows: $0.58,0.58,1.00,1.15$

Table 6 The thickness ratio of three types of plastic substrates (PMMA, PET, TPU) for soft sublayer with the minimum thickness of $10 \mu \mathrm{m}$ and the maximum thickness of $40 \mu \mathrm{m}$

\begin{tabular}{llll}
\hline Own thickness of plastic substrate & PMMA & PET & TPU \\
& $150 \mu \mathrm{m}$ & $125 \mu \mathrm{m}$ & $100 \mu \mathrm{m}$ \\
\hline SL-thickness/O-thickness (Max) & 0.266 & 0.32 & 0.4 \\
SL-thickness/O-thickness (Min) & 0.066 & 0.08 & 0.1 \\
\hline
\end{tabular}

a hard coating can be increased up to about twofold, but the improvement is marginal and is similar to the effect of the soft sublayer addition shown in (c) of Fig. 6.

Table 6 shows the thickness ratio of three types of plastic substrates (PMMA, PET, TPU) for soft sublayer with the minimum thickness of $10 \mu \mathrm{m}$ and the maximum thickness of $40 \mu \mathrm{m}$. According to the result of experiment, the improvement effect of scratch resistance was appeared when the thickness of the soft sublayer was $10 \%$ or more of the thickness of the plastic substrate. According to Fig. 6, the scratch resistance characteristics were the best when the plastic substrate was $150-\mu$ m-thick PMMA and the soft sublayer was $40 \mu \mathrm{m}$ thick. Therefore, the optimal thickness ratio of soft sublayer to improve scratch resistance when using PMMA as plastic substrate is [SLthickness/O-thickness $(\max )] \times 100 \%=26.6 \%$.

Since in the steel wool test, the steel wool bar is moving on the surface of the film, it has a dynamic feature. In this series of experiments, different moving speeds of steel wool bar were applied to the hard-coated PMMA film with and without a $40-\mu m$-thick soft sublayer. The steel wool test was carried out for each sample with two
Table 7 Effect of steel wool reciprocal abrasion speed

\begin{tabular}{lll}
\hline $\begin{array}{l}\text { Number of steel wool } \\
\text { reciprocal abrasions }\end{array}$ & 20 RPM $(27.6 \mathrm{~mm} / \mathrm{s})$ & 80 RPM $(107.7 \mathrm{~mm} / \mathrm{s})$ \\
\hline 20 & Scratch & Scratch \\
15 & Scratch & Scratch \\
10 & Scratch & Scratch \\
9 & Scratch & Scratch \\
8 & Scratch & Scratch \\
7 & Scratch & Scratch \\
6 & No Scratch & No Scratch \\
\hline
\end{tabular}

Specimens are hard-coated PMMA films without a soft sublayer

Table 8 Effect of steel wool reciprocal abrasion speed. Specimens are hard-coated PMMA films with a soft sublayer of $40 \mu \mathrm{m}$ thick

\begin{tabular}{lll}
\hline $\begin{array}{l}\text { Number of steel wool } \\
\text { reciprocal abrasions }\end{array}$ & 20 RPM $(27.6 \mathrm{~mm} / \mathrm{s})$ & 80 RPM $(107.7 \mathrm{~mm} / \mathrm{s})$ \\
\hline 120 & Scratch & Scratch \\
80 & Scratch & Scratch \\
70 & Scratch & No Scratch \\
60 & Scratch & No Scratch \\
50 & Scratch & No Scratch \\
40 & No Scratch & No Scratch \\
\hline
\end{tabular}

different reciprocal abrasion speeds at 20 RPM and 80 RPM. These speeds of 20 RPM and 80 RPM correspond to $27.6 \mathrm{~mm} / \mathrm{s}$ and $107.7 \mathrm{~mm} / \mathrm{s}$ in terms of linear speed, respectively. The results are summarized in Tables 7 and 8.

Generally, kinetic frictional force is not related to the moving speed of a moving object, so the frictional force would be the same even if the moving speed was different. In the case of the hard-coated PMMA film without the soft sublayer in Table 7, when the scratch resistance test was carried out at two speeds of 20 RPM and 80 RPM, scratches occurred when the number of steel wool reciprocal abrasions was seven times regardless of varying the speed. From this, it can be seen that film with a hard base such as PMMA has the same magnitude of kinetic frictional force irrespective of the moving speed.

If a soft sublayer is introduced under the hard-coated PMMA film, quite different features are observed. In Table 8, a 40- $\mu$ m-thick soft sublayer was added to the hardcoated PMMA film and the steel wool test was performed at two different speeds, 20 RPM and 80 RPM. In this case, the number of steel wool reciprocal abrasions at the time of scratch occurrence increased significantly at both moving speeds compared with the one without a soft sublayer. When the moving speed was 20 RPM, scratches occurred at steel wool reciprocal abrasions 50 times, and when the 
moving speed was 80 RPM, scratches occurred at steel wool reciprocal abrasions 80 times.

Since the frictional force has nothing to do with the moving speed of the steel wool, the possible reason could originate from other dynamic factors. One possible explanation would be the frequency dependency of damping in viscoelastic polymer. Tan $\delta$ is maximized at the glass transition temperature under a given frequency in any type of dynamic mechanical measurement. If the measuring frequency is increased, the glass transition temperature or the temperature of maximum energy dissipation will increase. Since the glass transition temperature of silicon is well below room temperature, the shift of moving speed from 20 to 80 RPM corresponds to a faster strain rate or higher frequency. Therefore, it can be envisioned that the value of $\tan \delta$ is on the uphill with the increase in moving speed. Then, the soft sublayer has a higher damping capability at 80 RPM than it does at 20 RPM.

Quite complex behavior has been observed when critical load was measured with different scratch strain rates [30]. When a $60^{\circ}$ conical probe was used, the critical load increased with the increase in the scratch strain rate, which is rather similar to the current observation. However, when a $90^{\circ}$ conical probe was employed, the trend was the opposite. The authors explained that such a difference comes from the difference in the reason for the fracture. For the $60^{\circ}$ cone, the critical load is sensitive to the stress exponent, while strain determines the fracture for the $90^{\circ}$ cone. Since the steel wool test is not a single-probe test, the situation becomes much more complex than a simple indenter scratch test. Moreover, the viscoelastic nature of polymer and the difference in behavior between being under tension and under compression makes interpretation even more complicated.

\section{Conclusions}

It has been shown that the employment of a low-modulus sublayer below the plastic subset can be a useful tool to improve scratch resistance without sacrificing surface hardness. In this study, the soft sublayer is made of crosslinked silicone compound and is applied to the back side of the hard-coated plastic substrate. The surface hardness does not show much variation with changes in the thickness of the soft sublayer, indicating that the presence of the soft sublayer does not greatly affect surface hardness.

On the other hand, the scratch resistance of the hardcoated plastic substrate could be greatly improved by adding the soft sublayer. Compared with a 0 - $\mu$ m-thick soft sublayer (without a soft sublayer), a $40-\mu \mathrm{m}$-thick soft sublayer results in an improvement in scratch resistance of 16 times when PMMA is selected as the base substrate.
Such an improvement seems to be the combined result of normal force variation by the deformation of the soft sublayer and damping capability of the soft sublayer.

However, when the hard PMMA substrate is replaced with softer PET and TPU, the improvement in scratch resistance become marginal, indicating that there is an optimal modulus ratio between the base substrate and soft sublayer.

One further thing to note is that scratch resistance depends on reciprocal abrasion RPM when it is measured by a steel wool test. In the current specimen and experimental setup, the scratch resistance at 80 RPM is much better than that measured at 20 RPM. It clearly seems to be related to the dynamic nature of polymer, but the detailed reason for such a difference is still a subject of further study.

\section{Compliance with ethical standards}

Conflict of interest The authors declare no conflict of interest.

\section{References}

1. Wang C, Shi K, Gross C, Julio MP, Lacerda Monica de Mesquita, Chung YW (2014) Toughness enhancement of nanostructured hard coatings: design strategies and toughness measurement techniques. J Surf Coat Technol 257:206-212

2. Son DH, Kim DS, Lee SH, Kim SH, Lee GD, Park SS (2012) Studies on the synthesis and characteristic of silica-PMMA nano hybrid material. Appl Chem Eng 23:53-58

3. Boersma A, Soloukhin VA, Brokken-Zijp JCM, De With G (2004) Load and depth sensing indentation as a tool to monitor a gradient in the mechanical properties across a polymer coating: a study of physical and chemical aging effects. J Polym Sci, Part B: Polym Phys 42:1628-1639

4. Yeo SM, Polycarpou AA (2013) Micromechanical properties of polymeric coatings. Tribol Int 60:198-208

5. Klapperich C, Komvopoulos K, Pruitt L (2001) Nanomechanical properties of polymers determined from nanoindentation experiments. J Tribol 123:624-631

6. Geng K, Yang F, Druffel T, Grulke EA (2005) Nanoindentation behavior of ultrathin polymeric films. Polymer 46:11768-11772

7. Wang M (2004) Nanoindentation of polymeric thin films with an interfacial force microscope. J Mech Phys Solids 52:2329-2354

8. Oliver WC, Pharr GM (1992) An improved technique for determining hardness and elastic modulus using load and displacement sensing indentation experiments. J Mater Res 7:1564-1583

9. Doerne MF, Nix WD (1986) A method for interpreting the data from depth-sensing indentation instruments. J Mater Res 1:601-609

10. Manideep G, Pankaj K, Monica K (2017) Review of mechanical characterization techniques for thin films used in flexible electronics. Crit Rev Solid State Mater Sci 42:129-152

11. Herve P, Christian G, Robert S (2010) Relationship between contact geometry and average plastic strain during scratch tests on amorphous polymers. Tribol Int 43:796-809 
12. Saha R, Nix WD (2002) Effects of the substrate on the determination of thin film mechanical properties by nanoindentation. Acta Mater 50:23-38

13. Tayebi N, Polycarpou AA, Conry TF (2004) Effects of substrate on determination of hardness of thin films by nanoscratch and nanoindentation techniques. J Mater Res 19:1791-1802

14. Yu N, Bonin WA, Polycarpou AA (2005) High-resolution capacitive load-displacement transducer and its application in nanoindentation and adhesion force measurements. Rev Sci Instrum 76(4):045109

15. Yeo CD, Polycarpou AA, Kiely JD, Hsia YT (2007) Nanomechanical properties of sub-10 nm carbon film overcoats using the nanoindentation technique. J Mater Res 22:141-151

16. Hay J (2014) Rapid mechanical properties of multi-layer film stacks, document No. 5991-4077EN, https://cal.equipment/doc/ HP_Agilent_Keysight/Rapid\%20Mechanical\%20Properties\%20 of $\% 20$ Multi-layer\%20Film\%20Stacks\%20-\%20Application $\% 20$ Note\%205991-4077EN\%20c20140318\%20\%5B8\%5D.pdf

17. VanLandingham MR, Chang NK, Wu YT, Li-Piin S, Jardret Vincent D, Shuo-Hung C (2004) Measurement approaches to develop a fundamental understanding of scratch and mar resistance. $J$ Coat Technol Res 1:257-266

18. Briscoe BJ (1998) Isolated contact stress deformations of polymers: the basis for interpreting polymer tribology. Tribol Int 31:121-126

19. Briscoe BJ, Evans PD, Pelillo E, Sinha SK (1996) Scratching maps for polymers. Wear 200:137-147

20. Briscoe BJ, Evans PD, Biswas SK, Sinha SK (1996) The hardnesses of poly(methylmethacrylate). Tribol Int 29:93-104

21. Briscoe BJ, Pelillo E, Sinha SK (1996) Scratch hardness and deformation maps for polycarbonate and polyethylene. Polym Eng Sci 36:2996-3005
22. Briscoe BJ, Pelillo E, Sinha SK (1997) Characterisation of the scratch deformation mechanisms for poly(methylmethacrylate) using surface optical reflectivity. Polym Int 43:359-367

23. Chu J, Xiang C, Sue H-J, Hollis RD (2000) Scratch resistance of mineral-filled polypropylene materials. Polym Eng Sci 40:944-955

24. Xiang C, Sue H-J, Chu J, Coleman B (2001) Scratch behavior and material property relationships in polymers. J Polym Sci Part B Polymer Phys 39:47-59

25. Gauthier C, Schirrer R (2000) Time and temperature dependence of the scratch properties of poly(methylmethacrylate) surfaces. J Mater Sci 35:2121-2130

26. Gauthier C, Lafaye S, Schirrer R (2001) Elastic recovery of a scratch in a polymeric surface: experiments and analysis. Tribol Int 34:469-479

27. Mi L, Ling H, Shen W, Ryntz R, Wichterman B, Scholten A (2006) Some complementary scratch resistance characterization methods. J Coat Technol Res 3:249-255

28. Humood M, Chowdhury S, Song Y, Tzeng P, Grunlan JC, Polycarpou AA (2016) Nanomechanical behavior of high gas barrier multilayer thin films. ACS Appl Mater Interfaces 8:11128-11138

29. Sujan $E$, Bin Wadud detection of adhesive layer between polyester films using DMA, http://www.tainstruments.com/pdf/liter ature/RH087_Adhesive_layer_between_films.pdf

30. Vincent J, Morel MP (2003) Viscoelastic effects on the scratch resistance of polymers: relationship between mechanical properties and scratch properties at various temperatures. Prog Organ Coat 48:322-331

Publisher's Note Springer Nature remains neutral with regard to jurisdictional claims in published maps and institutional affiliations. 